\title{
Neural Network PID Algorithm for a Class of Discrete-Time Nonlinear Systems
}

\author{
https://doi.org/10.3991/ijoe.v14i02.7914 \\ Huifang Kong $\left({ }^{\varpi}\right)$, Yao Fang \\ Hefei University of Technology, Hefei, P.R.China \\ konghuifang@163.com
}

\begin{abstract}
The control of nonlinear system is the hotspot in the control field. The paper proposes an algorithm to solve the tracking and robustness problem for the discrete-time nonlinear system. The completed control algorithm contains three parts. First, the dynamic linearization model of nonlinear system is designed based on Model Free Adaptive Control, whose model parameters are calculated by the input and output data of system. Second, the model error is estimated using the Quasi-sliding mode control algorithm, hence, the whole model of system is estimated. Finally, the neural network PID controller is designed to get the optimal control law. The convergence and BIBO stability of the control system is proved by the Lyapunov function. The simulation results in the linear and nonlinear system validate the effectiveness and robustness of the algorithm. The robustness effort of Quasi-sliding mode control algorithm in nonlinear system is also verified in the paper.
\end{abstract}

Keywords-dynamic linearization model, quasi-sliding mode control, nonlinear system, neural network

\section{Introduction}

With increasing demands on the improvement of the precision control, the control problem of nonlinear system is attracting more and more attention[1]. Especially, the robustness problem of nonlinear control system model has gradually become an important topic in control theory. In general, control algorithms of nonlinear system mainly contain the neural network control, fuzzy logic control, the sliding mode control and model free adaptive control (MFAC).

From universal approximation theory, a single hidden layer neural network can approximate any nonlinear function to any prescribed accuracy if sufficient hidden neurons are provided[2]. However, the training examples are usually much larger than the hidden nodes, and selecting the appropriate number of hidden nodes is the key factor which determining the error of the prediction model. And the algorithm of the control system can't ensure the global optimal value of weight[3]. The fuzzy logic system can also approximate any nonlinear function to any prescribed accuracy, and it has the advantages in dealing with the time-delay, time-varying, multi input single output nonlinear system[4]. Especially, the combination between fuzzy control and PID 
control algorithm, the objective of improved control algorithm is to eliminate the steady state error, and the algorithm can also improve the control accuracy and stationary performance. However, the establishment of fuzzy rules is lack of the systemic approach, and it has to rely on the engineering experiments[5]. Sliding mode variable structure control (SMC) has good robustness to perturbation and external disturbance in sliding mode, so it becomes one of the effective methods to deal with nonlinear systems with various uncertainties[6]. However, the variable structure control will be the quasi-sliding mode control for nonlinear system. Especially, with the application of the computer, the properties, stability and reaching conditions of sliding mode variable structure are changed; and variable structure control method of continuous time systems can not be directly applied to the discrete time system. Hence, the quasisliding mode control has important theoretical value and practical significance to study the variable structure control method for discrete-time nonlinear systems. In recent years, the theory and design of variable structure control for discrete time systems are gradually increasing, and adaptive variable structure control method attracts many scholars because of its advantages of adaptive control and variable structure control[7]. And, in order to solve the disturbance problem in the system, the upper bound of uncertainty is adopted; it can also express that the disturbance problem is solved by the worst situation. It is no doubt that the dynamic performance and robustness of system can be worse. However, the boundary parameters are difficult to obtain in real control system. It is all known that the modeling of nonlinear systems is timeconsuming and laborious, and it is very difficult to build reliable models for many nonlinear systems, and the cost of modeling is very high. The Model Free Adaptive Control (MFAC) is proposed to build the dynamic linearization model of nonlinear system, the algorithm is based on the theory of pseudo-partial-derivative (PPD), and the controller just uses the input and output information[8-9].

The paper combines the dynamic linearization ideology of MFAC and quasisliding mode control algorithm to build a precise model and the neural network is designed to obtain the optimal control law based on the above model. The control algorithm has the following advantages, (1) a better robustness; (2) a better tracking performance of system.

\section{System identification and control law of the nonlinear system}

\subsection{Dynamic linearization of the nonlinear system}

Considering the following unknown discrete-time SISO system

$$
y(k+1)=\Gamma\left(y(k), \ldots, y\left(k-n_{y}\right), u(k), \ldots, u\left(k-n_{u}\right)\right)
$$

where $\Gamma(\cdot)$ is an unknown nonlinear difference equation representing the plant dynamics, $u(k) \in \mathfrak{R}^{u}$ and $y(k) \in \mathfrak{R}^{y}$ are measurable scalar input and output, $n_{y}$ and $n_{u}$ is the unknown order of the system. The system (1) is the normal NARMA model. 
The following assumptions are made about the model (1):

A1: The system model is observable and controllable. This is the basic requirement for designing controller parameters.

A2: The partial derivative of $\Gamma(\cdots)$ which is with respect to the input parameters $u(k)$ is continuous. Assumption A2 is a typical condition of control system design for general nonlinear system.

A3: The system generalized Lipschitz, this is $\left|y\left(k_{1}+1\right)-y\left(k_{2}+1\right)\right| \leq b\left|u\left(k_{1}\right)-u\left(k_{2}\right)\right|$ for any $k_{1}$ and $k_{2}, k_{1} \neq k_{2}, k_{1}, k_{2} \geq 0$ and $u\left(k_{1}\right) \neq u\left(k_{2}\right)$.Assumption A2 poses a limitation on the rate of change of the system output permissible before the control law to be formulated is applicable.

Theorem 1 For the nonlinear system (1) satisfying the assumptions A1-A3, then there must exist a time-varying parameter $\phi(k)$, called pseudo-partial-derivative $(\mathrm{PPD})^{[10]}$. If $\Delta \mathrm{u}(k) \neq 0$, the system (1) can be described as the following model.

$$
\bar{y}(k+1)=\bar{y}(k)+\phi(k) \Delta u(k)
$$

Where, $\bar{y}(k)$ is the estimation output of system model, $\phi(k) \leq b$ and $b$ is the positive constant.

Remark: In order to make the condition $\Delta u(k) \neq 0$ in (2) be satisfied, and meanwhile to make the parameter estimation algorithm has stronger ability in tracking time-varying parameter, a reset algorithm has been added into this MFAC scheme as follows :

if $|\hat{\phi}(k)| \leq \varepsilon$ or $\operatorname{sign}(\hat{\phi}(k)) \neq \operatorname{sign}(\hat{\phi}(1)) \quad$ or $|\Delta \mathrm{u}(k-1) \leq \varepsilon|$, then $\hat{\phi}(k)=\hat{\phi}(1)$. Where $\varepsilon$ is a small positive constant, and $\hat{\phi}(1)$ is the initial value of $\hat{\phi}(k)$.

Considering $\phi(k)$ is a time-varying parameter, only the input and output data can be used to get the optimal solution. The objective function can be designed in the following way :

$$
J(\phi(k))=|\bar{y}(k)-\bar{y}(k-1)-\phi(k-1) \Delta u(k-1)|^{2}+\mu|\phi(k)-\hat{\phi}(k-1)|^{2}
$$

where $\mu>0$ is weight factor, and $\hat{\phi}(k-1)$ is the estimated value of the delay time.

Getting the partial derivative about the $\phi(k)$,we can get the optimal estimate value

$$
\hat{\phi}(k)=\hat{\phi}(k-1)+\frac{\eta \Delta u(k-1)}{\mu+\Delta u(k-1)^{2}} *(\bar{y}(k)-\bar{y}(k-1)-\hat{\phi}(k-1) \Delta u(k-1))
$$

where $\eta \in(0,1]$ is the step factor. 


\subsection{Model error estimation using Quasi-sliding mode algorithm}

In the last section, the dynamic linearization model (DL model) is designed to represent the nonlinear system. But the model error still exists; in this section, we use the Quasi-sliding mode control algorithm to estimate the model error. Define the estimation error of dynamic linearization model $\bar{e}(k)$ :

$$
\bar{e}(k)=y(k)-\bar{y}(k)
$$

where $\mathrm{y}(\mathrm{k})$ and $\bar{y}(k)$ are the real output and dynamic linearization model output respectively. Define the function of sliding mode surface $s(k)^{[11]}$

$$
s(k)=\mathrm{c}^{T} E(k)=\bar{e}(k)+c_{0} \bar{e}(k-1)
$$

where $\mathrm{c}^{T}=\left[1, c_{0}\right]^{T}, c_{0}$ is a positive value. Adopting discrete-time sliding mode reaching-law

$$
\begin{gathered}
(s(k+1)-s(k)) / T=-q s(k)-\varepsilon \operatorname{sgn}(s(k)) \\
s(k+1)=(1-q T) s(k)-\varepsilon T \operatorname{sgn}(s(k))
\end{gathered}
$$

where $\varepsilon>0, \quad 1>q T>0$ and $T>0$ is sampling period. Rewritten (7) as

$$
s(k+1)=(1-q T) s(k)-\varepsilon T \operatorname{sgn}(s(k))=\mathrm{c}^{T} E(k+1)
$$

According to the formula (6)-(9), we can get the estimation of the model error $\bar{e}(k+1)$ at the next sampling time:

$$
\bar{e}(k+1)=(1-q T) s(k)-\varepsilon T \operatorname{sgn}(s(k))-c_{o} \bar{e}(k)
$$

In summary, the discrete-time SISO model can be represented in the formula (11):

$$
\tilde{y}(k+1)=\bar{y}(k+1)+\bar{e}(k+1)
$$

where $\tilde{y}(k)$ is the estimation value of system model after the addition of Quasisliding mode algorithm. $\bar{e}(k+1)$ and $\bar{y}(k+1)$ represent the estimation error and output of the dynamic linearization model in the next sample time, respectively. The addition of QSMC is to predict the estimation error in advance and feed back to the neural network controller, therefore, the system can converge in a shorter time.

\subsection{Control law design based on the artificial neural network}

In order to get the optimal control law, we adopt the following structure of the nonlinear system. NNC is a neural network controller. DL model represents the dynamic 
linearization model in form of (2), and QSMC is the Quasi-sliding mode controller in section 2.2. The structure of completed control algorithm is shown in the figure 1.

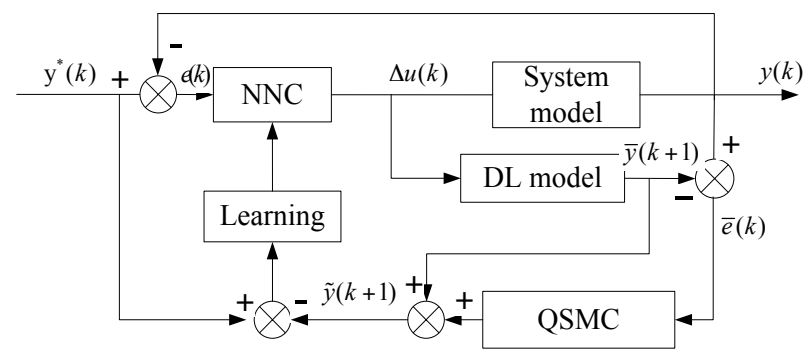

Fig. 1. the completed control structure of the nonlinear system

The structure of NNC. The NNC (neural network controller) is represented by the following structure, which is the three-layer neural network. The first layer of NNC is input layer, the input data $X(k)=[e(k), e(k-1), e(k-2)]$, where $e(k)$ represents the tracking error between real output and expected output at sample time $k$.

$$
e(k)=y^{*}(k)-y(k)
$$

where $y^{*}(k), y(k)$ represent the desired output and real output respectively.

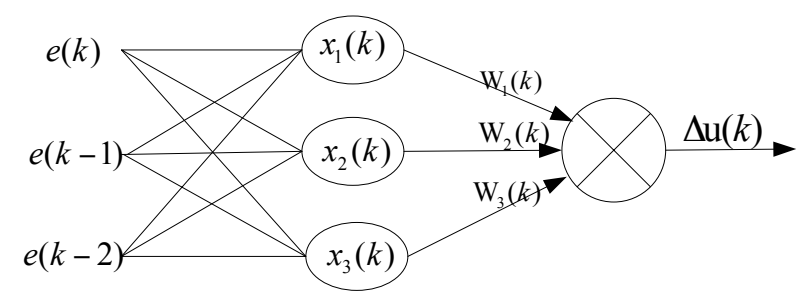

Fig. 2. The inner structure of NNC

The second layer is the hidden layer which contains three neural units; the response of a neural unit is as follows.

$$
\left\{\begin{array}{l}
x_{1}(k)=e(k) \\
x_{2}(k)=\Delta e(k)=e(k)-e(k-1) \\
x_{3}(k)=\Delta^{2} e(k)=e(k)-2 e(k-1)+e(k-2)
\end{array}\right.
$$

The output of neural network controller

$$
\Delta u(k)=w_{1} x_{1}(k)+w_{2} x_{2}(k)+w_{3} x_{3}(k)
$$


where $\left\{w_{i}\right\}, i=1,2,3$ means the weight of neural network, when the appropriate value of the $w_{i}$ is selected, the controller usually can have a good performance. $\Delta u(k)$ is variable rate of control law. $u(k)=u(k-1)+\Delta u(k)$ is the control law at the sampling time $k$. So the NNC has the structure of neural network PID controller.

The weight of the neural network controller $\left\{w_{i}\right\}, i=1,2,3$ can be trained by the optimal objective function $J_{c}=\frac{1}{2}\left[y^{*}(k)-\tilde{y}(k)\right]^{2}$. So

$$
\Delta w_{i}(k)=-\lambda \frac{\partial J_{c}(k)}{\partial w_{i}}=\lambda\left[y^{*}(k)-\tilde{y}(k)\right] \hat{\phi}(k) x_{i}(k)
$$

where $\lambda>0$ means the learning rate of neural network controller, and $i=1,2,3$. $w_{i}(k)=w_{i}(k-1)+\Delta w_{i}(k)$ means the weight of neural network controller at the sampling time $k$.

\section{The stability and convergence of control algorithm}

In order to obtain the convergence and stability of the controller of nonlinear system, another assumption about the controlled system should be made.

A4: The PPD satisfies $\phi_{c}(k)>\vartheta>0$ or $\phi_{c}(k)<-\vartheta$, and $\vartheta$ is a small positive constant. Without the loss of generality, it is assumed in this paper.

Theorem 1: The nonlinear system (1) satisfies Assumption 1-3, the estimation algorithm of model adopts the formula (5-6). The control rate adopts formula (15). If the expected output of nonlinear system is bounded, and the estimation of model is stable, and the control algorithm is stable. And the real output and input is bounded.

Proof :

1) Time-varying parameter $\hat{\phi}(k)$ is bounded.

Considering the length of article, the paper don't give the detailed proof, the detailed process is stated in the paper[10].

2) The convergence of the nonlinear system model

Define Lyapunov function

$$
V(k)=[s(k)]^{2}+\delta^{2}
$$

where $\delta$ is a small positive const to guarantee $V(k)$ which is a positive function. According to the reaching-law of continuous sliding mode control, the reaching law of discrete-time sliding mode control can be written as ${ }^{[12]}$.

$$
\Delta V(k)=V(k+1)-V(k)<0
$$




$$
\begin{aligned}
& \Delta V(k)=V(k+1)-V(k)=(s(k+1))^{2}-(s(k))^{2} \\
& =[(1-q T) s(k)-\varepsilon T \operatorname{sgn}(s(k))]^{2}-(s(k))^{2} \\
& =-q T(2-q T)-2 \varepsilon T(1-q T)|s(k)|
\end{aligned}
$$

Since $1>q T>0, \varepsilon, q, T>0$, hence $\Delta V \leq 0$.

According to the Lyapunov stability theorem, the identification model is stable. On the promise of the given initial value, $V(0)$ is bounded, using the control law (14), the estimation of model error $\bar{e}(k)$ tends to zero, this is $\lim _{k \rightarrow \infty}|y(k)-\tilde{y}(k)|=|y(k)-\bar{y}(k)|=0$.

3) The convergence of control algorithm

According to the approach ability of neural network, the network can optimize the parameters of controller by the error back-propagation way, so the output of controller can approach the desired output[13]. Define global Lyapunov function

$$
\begin{gathered}
V_{g}(k)=0.5 *(e(k))^{2} \\
\Delta V_{g}(k)=0.5 *(e(k)+\Delta e(k))^{2}-0.5 *(e(k))^{2} \\
=e(k) * \Delta e(k)+0.5 *(\Delta e(k))^{2}
\end{gathered}
$$

As we all know, $e(k)$ is the function about the $\left\{w_{i}\right\}$, it also $e(k) \rightarrow f\left(w_{1}, w_{2}, w_{3}\right)$. According to the principle of calculus, we can get the following expression.

$$
\begin{aligned}
\Delta e(k) & =\sum_{1}^{3} \frac{\partial e(k)}{\partial w_{i}} * \Delta w_{i}=\sum_{1}^{3} \frac{\partial\left(y^{*}(k)-\tilde{y}(k)\right)}{\partial w_{i}} * \Delta w_{i} \\
& =-\sum_{i=1}^{3} \frac{\partial(\bar{y}(k-1)+\hat{\phi}(k) * \Delta u(k))}{\partial w_{i}} * \Delta w_{i} \\
& =-\hat{\phi}(k) \sum_{i=1}^{3} \frac{\partial(\Delta u(k))}{\partial w_{i}} * \Delta w_{i} \\
& =-\lambda(\hat{\phi}(k))^{2}\left[y^{*}(k)-\tilde{y}(k)\right] * \sum_{i=1}^{3}\left(x_{i}(k)\right)^{2}
\end{aligned}
$$

Due to the estimation model is convergence, so the real output $y(k)$ tends to equal to the estimation value $\tilde{y}(k)$. So we can get the following conclusion. 


$$
\Delta e(k) \cong-\lambda(\hat{\phi}(k))^{2}\left[y^{*}(k)-y(k)\right] * \sum_{i=1}^{3}\left(x_{i}(k)\right)^{2}
$$

According to the formula (20) and (22),

$$
\begin{aligned}
& \Delta V_{g}(k)=\Delta e(k)(e(k)+0.5 * \Delta e(k)) \\
& =-\lambda(\hat{\phi}(k))^{2}\left[y^{*}(k)-y((k))\right]^{2} \sum_{i=1}^{3}\left(x_{i}(k)\right)^{2}\left[1-0.5^{*} \lambda(\hat{\phi}(k))^{2} * \sum_{i=1}^{3}\left(x_{i}(k)\right)^{2}\right]
\end{aligned}
$$

If $\frac{1}{2} \lambda(\hat{\phi}(k))^{2} * \sum_{i=1}^{3}\left(x_{i}(k)\right)^{2} \leq 1$, hence $\Delta V_{g}(k) \leq 0$, according to the principle of Lyapunov stability, the control system is stable.

Overall, the maximal tracking error of nonlinear system can be expressed $\operatorname{Max}(e(k))=\max \left|y^{*}(k)-y(k)\right|<\xi$. In the range of allowable error, the control system has a global convergence.

4) The boundness of real output and input

$\operatorname{Max}(e(k))=\max \left|y^{*}(k)-y(k)\right|<\zeta, e(k)$ is bounded, and the desired output is bounded. So the real output is bounded. Since the time-varying parameter $\hat{\phi}(k)$ is bounded, and $|u(k)| \leq \lambda_{1} \operatorname{Max}(|y(k)|)+\lambda_{2}$, and $\lambda_{1}, \lambda_{2}$ is a const. Hence $u(k)$ is also bounded.

Clearly, the Theorem 1 can be proved.

\section{Simulation results}

In this section, the two simulations are used to show the effectiveness and robustness of the control algorithm.

1. The convergence and superiority of algorithm compared with the traditional PID algorithm in linear system.

2. The convergence and robustness of control system in nonlinear system.

\subsection{The convergence and robustness of algorithm in linear system}

The convergence of algorithm in linear system. As we all known, the traditional PID algorithm is the most important control algorithm in linear system. The output of the incremental PID algorithm is

$$
\Delta u(k)=k_{p} \Delta e(k)+k_{i} e(k)+k_{d} \Delta^{2}(e(k))
$$


In order to verify the superiority compared with the traditional PID algorithm, the discrete-time linear system is defined as follows

$$
y(k)=0.8 * y(k-1)-0.5 y(k-2)+0.5 u(k-1)
$$

where $k$ is a positive integer, and the desired output be written as (26)

$$
y^{*}(k)=1 \quad k<40
$$

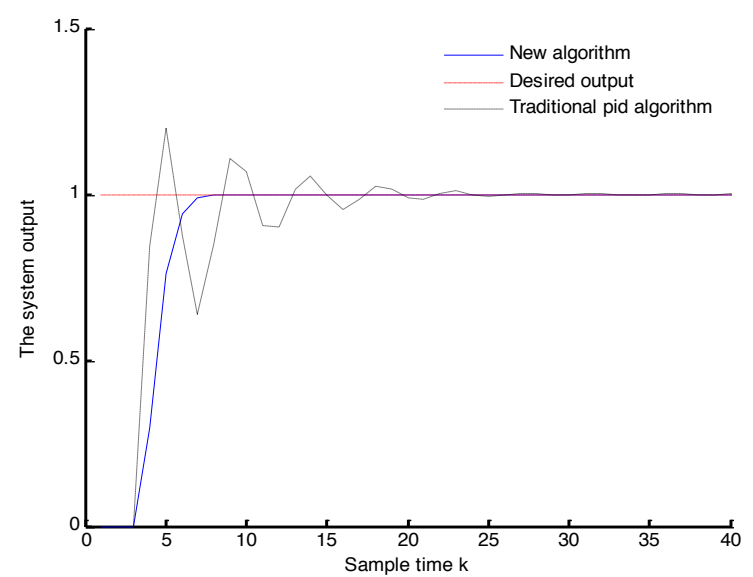

Fig. 3. The tracking performance compared with traditional PID in linear system

The simulation diagram is showed in figure 3, as we can see, if the sampling time is less than 3 , the controller needs to collect the error information of the tracking system, so the real output equal to zero; After three sample time, the control system can be stable in the finite time; the real output can track the desired output perfectly and the error of tracking can be in allowable range. The simulation results show the new algorithm can depress the overshoot compared with the traditional PID algorithm, decrease the adjustment time and get the satisfactory tracking performance.

The robustness of algorithm in linear system. The robustness of system is the important index in control systems, it refers to the perturbation of system parameters or system structure, the system still can maintain the characteristics, like convergence or stability. In order to verify the robustness of linear system compared with the traditional PID algorithm, the simulation model is as follows, the parameters perturbation happens at sample time $k=30$.

$$
y(k)=\left\{\begin{array}{l}
0.8 * y(k-1)-0.5 y(k-2)+0.5 u(k-1) k<30 \\
0.8 * y(k-1)-0.8 y(k-2)+0.5 u(k-1) k>=30
\end{array}\right.
$$




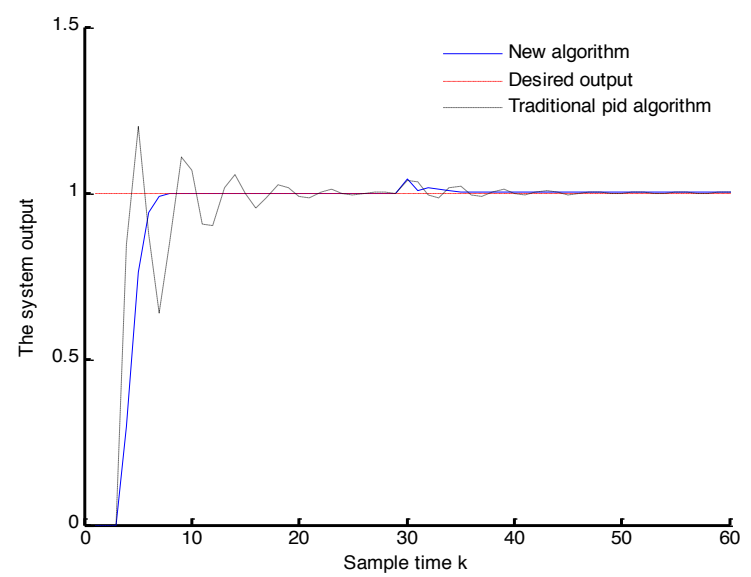

Fig. 4. The robustness of algorithm compared with traditional PID algorithm in linear system

As we can see in the figure 4, when the structure parameters of system change at the sample time $k=30$, the new algorithm still has the better tracking performance, and the tracking error can be in the range of allowable at the finite time. And the adjustment time and adjustment amplitude of new algorithm can be smaller during the period of the system parameters perturbation compared with traditional PID algorithm.

\subsection{The convergence and robustness of nonlinear system}

The convergence and superiority compared with the MFAC algorithm. MFAC (model free adaptive control), as we know, has the good performance in the nonlinear control system. So, in order to verify the superiority, we compare with the MFAC algorithm. The discrete-time nonlinear system

$$
y(k)=\frac{y(k-1)}{1+(y(k-1))^{2}}+(u(k))^{3}
$$

where $u(k)$ is the control variable of controller, $y(k)$ and $y(k-1)$ are the current and the one step delayed outputs, respectively. And the desired output of the system is as follows:

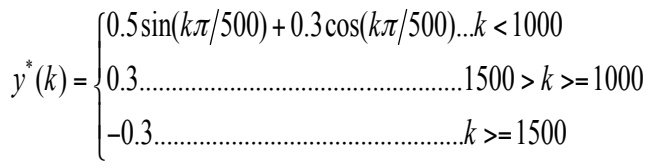




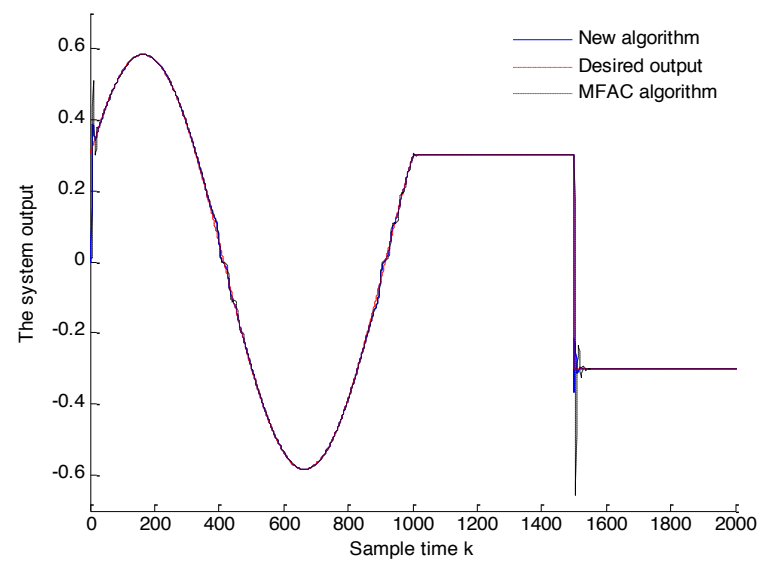

(a) the comparative figure between new algorithm and MFAC algorithm

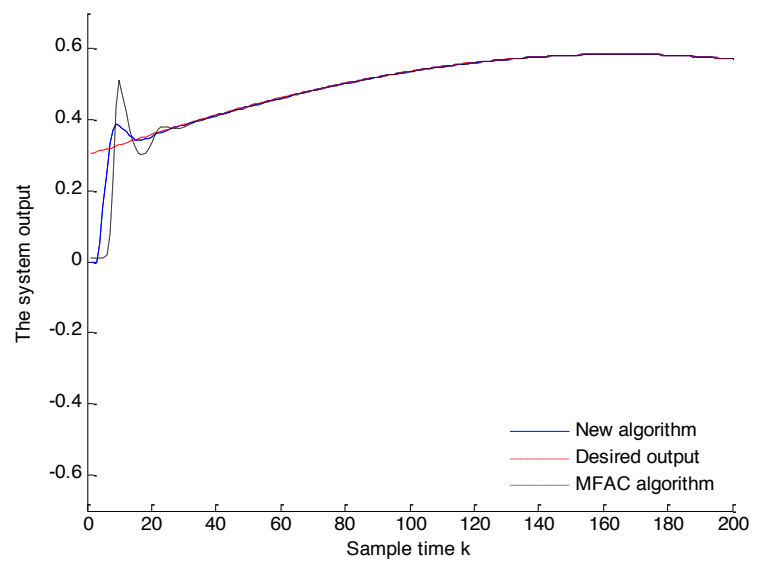

(b) Local enlarged figure of simulation results

Fig. 5. The superiority of control system compared with the MFAC algorithm

As we can see from the figure 5(a), the tracking error of system can be ignored in the range of error allowed except the sudden change of desired output. The simulation result validates the convergence of the algorithm and the stability of the system identification in nonlinear system. As shown in the local enlarged figure 5(b), the MFAC and new algorithm can track the desired output after the several step times. And the adjustment time and adjustment amplitude of the new algorithm have a great improvement compared with the MFAC algorithm.

The robustness of control system. In order to verify the robustness of the algorithm, the paper adopts the same control parameters to validate the robustness of the 
new control algorithm; the tracking performance is shown in the figure 6 . The simulation model is adopted (30), and the desired output is as the form of (29).

$$
y(k)=\left\{\begin{array}{l}
\frac{y(k-1)}{1+(y(k-1))^{2}}+(u(k))^{3} \ldots \ldots \ldots \ldots . . . k<1200 \\
\frac{y(k-1)}{1+(y(k-1))^{2}}+(u(k))^{2} \ldots . .1300>k>=1200 \\
\frac{0.8^{*} y(k-1)}{1+(y(k-1))^{2}}+(u(k))^{3} \ldots \ldots \ldots . . . k>=1300
\end{array}\right.
$$

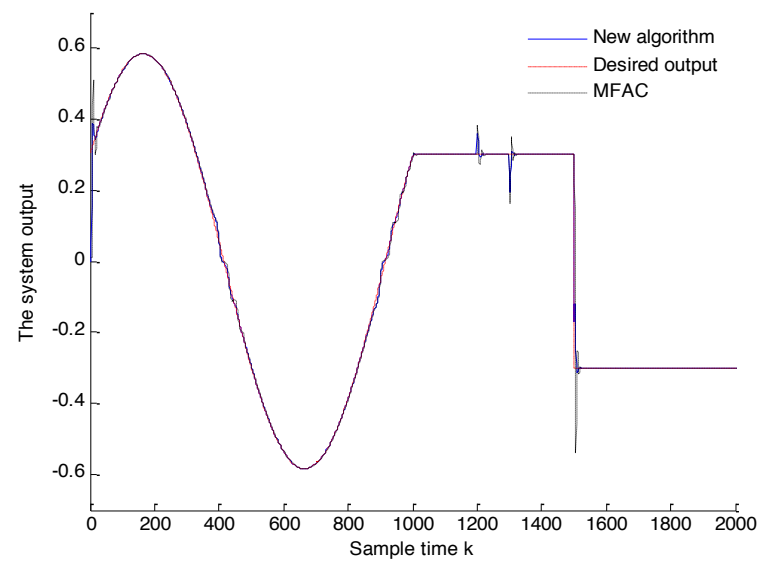

(a) The robustness analysis figure in nonlinear system

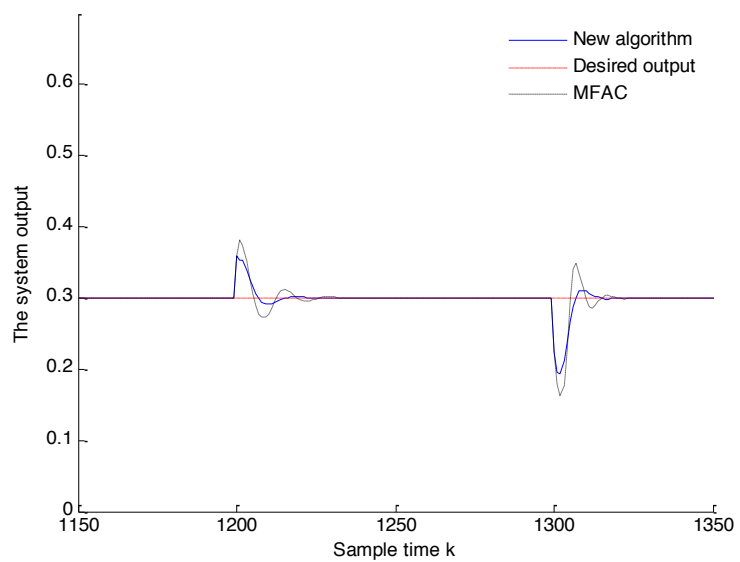

(b) The local enlarged figure between new algorithm and MFAC algorithm

Fig. 6. The robust analysis in the nonlinear system 
As we can see in the figure 6 , when the structure parameters of system change at the sampling time $k=1200, k=1300$, the algorithm still has the better tracking performance, and the tracking error can be in the range of allowable at the finite time. From the local enlarged figure 6(b), we can see that the adjustment time and adjustment amplitude of the new algorithm is less than the MFAC algorithm.

\section{Conclusion}

In this paper, a new dynamic linearization model about the Quasi-Sliding Mode algorithm was proposed for identification of complex nonlinear system. A neural network PID controller is designed to get the optimized control rate based on the above model. The advantages of algorithm are as follows:

1. better robustness

2. better tracking performance

3. less adjustment time and amplitude.

And the global stability of the system is proved by the way of the Lyponou function and Matlab simulation.

\section{Acknowledgment}

The work is supported by the National Science and technology support program (NO 2014BAG06B02) and Fundamental Research Funds for the Central Universities (No 2014HGCH0003).

\section{$7 \quad$ References}

[1] Ding, Feng. "Hierarchical multi-innovation stochastic gradient algorithm for Hammerstein nonlinear system modeling." Applied Mathematical Modelling 37.4 (2013): 1694-1704. https://doi.org/10.1016/j.apm.2012.04.039

[2] Nigam, Vivek Prakash, and Daniel Graupe. "A neural-network-based detection of epilepsy." Neurological Research 26.1 (2004): 55-60. https://doi.org/10.1179/01616410 4773026534

[3] Wang, Tong, Huijun Gao, and Jianbin Qiu. "A combined adaptive neural network and nonlinear model predictive control for multirate networked industrial process control." IEEE Transactions on Neural Networks and Learning Systems 27.2 (2016): 416-425. https://doi.org/10.1109/TNNLS.2015.2411671

[4] Li, Hongyi, et al. "Control of nonlinear networked systems with packet dropouts: interval type-2 fuzzy model-based approach." IEEE Transactions on Cybernetics 45.11 (2015): 2378-2389. https://doi.org/10.1109/TCYB.2014.2371814

[5] Liu, Yan-Jun, Shaocheng Tong, and CL Philip Chen. "Adaptive fuzzy control via observer design for uncertain nonlinear systems with unmodeled dynamics."IEEE Transactions on Fuzzy Systems21.2 (2013): 275-288. https://doi.org/10.1109/TFUZZ.2012.2212200 
[6] Hou, Yi-You, and Zhang-Lin Wan. "Robust Stability for Nonlinear Systems with TimeVarying Delay and Uncertainties via the Quasi-Sliding Mode Control." International Journal of Antennas and Propagation 2014 (2014). https://doi.org/10.1155/2014/897179

[7] Ma, Haifeng, Jianhua Wu, and Zhenhua Xiong. "Discrete-time sliding-mode control with improved quasi-sliding-mode domain." IEEE Transactions on Industrial Electronics 63.10 (2016): 6292-6304. https://doi.org/10.1109/TIE.2016.2580531

[8] Zhu, Yuanming, and Zhongsheng Hou. "Data-driven MFAC for a class of discrete-time nonlinear systems with RBFNN." IEEE transactions on neural networks and learning systems 25.5 (2014): 1013-1020. https://doi.org/10.1109/TNNLS.2013.2291792

[9] Ma, Ping, et al. "Main steam temperature control system based on MFAC." Electric Power Science and Engineering 1 (2006): 006.

[10] Cheng, Zhihui, Zhongsheng Hou, and Shangtai Jin. "MFAC-based balance control for freeway and auxiliary road system with multi-intersections." Control Conference (ASCC), 2015 10th Asian. IEEE, 2015.

[11] Han, Yueqiao, Yonggui Kao, and Cunchen Gao. "Robust sliding mode control for uncertain discrete singular systems with time-varying delays and external disturbances." Automatica 75 (2017): 210-216. https://doi.org/10.1016/j.automatica.2016.10.001

[12] Sarpturk, S. Z., Yorgo Istefanopulos, and Okyay Kaynak. "On the stability of discrete-time sliding mode control systems." IEEE Transactions on Automatic Control 32.10 (1987): 930-932. https://doi.org/10.1109/TAC.1987.1104468

[13] Mukhopadhyay, Snehasis, and Kumpati S. Narendra. "Disturbance rejection in nonlinear systems using neural networks." IEEE Transactions on Neural Networks 4.1 (1993): 6372. https://doi.org/10.1109/72.182696

[14] Chandrasena Premawardhena, N., ICT in the foreign language classroom in Sri Lanka: A journey through a decade. 10th World Conference on Computers in Education (WCCE 2013), Nicolaus Copernicus University, July 2-5 2013, Torun, Poland.pp 223-224

\section{Authors}

Huifang Kong is the Professor and the Director of the Research Center of Automotive Electronics and Control Technology in Hefei University of Technology, No 193, Tunxi Road, Hefei, China, 230009. Her research interests including Control Engineering, Industrial Automation, and Dynamics and Control. (konghuifang@163.com).

Yao Fang is a PH.D student in the Automotive Electronics and Control Research Center in Hefei University of Technology, Majoring in modeling and control of complex nonlinear systems, Energy management of hybrid electric vehicle and the behavior decision of drivers.

Article submitted 31 October 2017. Resubmitted 11 December 2017. Final acceptance 06 February 2018. Final version published as submitted by the authors. 